\title{
Pressure Injury Related to Friction and Shearing Forces in Older Adults
}

\author{
Vianna V. Broderick, Linda J. Cowan* \\ James A. Haley Veterans Hospital and Clinics, 13000 Bruce B. Downs Blvd, Tampa, FL 33612
}

\section{Article Info}

Article Notes

Received: May 21, 2021

Accepted: July 12, 2021

\section{*Correspondence:}

*Dr. Linda J. Cowan, James A. Haley Veterans' Hospital and Clinics, 13000 Bruce B. Downs Blvd, Tampa, FL 33612; Email: Linda.Cowan@va.gov

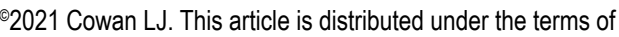
the Creative Commons Attribution 4.0 International License.

\section{Introduction}

The scientific literature describes many physiologic changes to the human body with age. These changes have a significant impact on the potential harm from pressure, friction, and shearing forces on the skin. ${ }^{1}$ Prolonged stay or immobility on surfaces such as beds and chairs are known to increase the risk of pressure injuries (PIs). ${ }^{2}$ Most PI prevention strategies target pressure force risk reduction through mechanical offloading, repositioning, and pressure redistribution support surfaces. ${ }^{3}$ However, the movement of repositioning may increase friction and shearing forces that may lead to PIs if not done properly especially in the older population. ${ }^{4}$ Diminished skin integrity and increased skin fragility with aging highlight the need for PI prevention strategies to address friction and shear risk reduction. ${ }^{2,4}$ This mini review of the literature provides a brief summary of PI injury related to friction and shearing forces in older adults.

\section{Aging Skin and Pressure Injury}

The process of aging skin is well documented in many publications. Research demonstrates that younger skin composition is very organized with histological findings of rete ridges effacement at the dermal-epidermal junction, complex elastic fiber arrays, and basketweave fibrillar collagen leading to resistance of skin deformation. ${ }^{5,6}$ Aging impacts multiple microcellular processes involved in cytoskeletal tissue maintenance including those regulating immune function, metabolism, hemostasis, and skin microbiome composition leading to functional changes. ${ }^{1}$ Skin over time diminishes its structural organization and ability to act as a barrier to microbes, toxins, sunlight, and radiation leading to PI risk with prolonged healing time. ${ }^{1,5,7,8}$ Skeletal tissue changes with aging include diminished muscle mass, decreased epidermal thickness, flattening of epidermal rete ridges and dermal papillae, loss of subcutaneous fat, and reduced skin elasticity. ${ }^{9}$ The skin dermal matrix also becomes thinner, crosslinked, and fragmented as people age. ${ }^{1,9}$ The reduction of subcutaneous fat among older adults contributes to saggy skin and wrinkles along with vulnerability to injury from mechanical stress over bony prominences. ${ }^{9} \mathrm{~A}$ tensile load causes the dermal collagen fibers beneath to straighten in the direction of the pressure and thus weakens the skin structure with increased risk of injury. ${ }^{9}$ Skin microstructure influences friction and shear particularly at the superficial layers where the outer most layer of the skin, stratum corneum, is typically 15 to 20 cell thickness of keratinized epithelial cells. ${ }^{10}$ Humidity and moisture 
can soften the stratum corneum, which increases contact, adhesion, friction, and deformation leading to more risk of blisters, skin tears, and superficial PIs. ${ }^{10}$

To summarize pressure-related tissue damage, PIs are identified by their appearance into four main stages and two additional categories. Stage 1 PI is non-blanchable erythema of intact skin over a bony prominence. ${ }^{2,11,12}$ Stage 2 PI involves partial thickness loss of the epidermis and possibly dermis or may appear as a clear fluid blister. 2,11, 12 Stage $3 \mathrm{PI}$ is full thickness skin loss that involves subcutaneous fat, and Stage 4 PI is full thickness skin loss that extends to muscle, tendon, ligament, cartilage, or bone. ${ }^{2,11,12}$ Unstageable PIs are wounds covered with nonviable tissue that obscure the accurate depth of injury for staging. ${ }^{2,12}$ Deep tissue injury (DTI) involves tissue changes at the bone-muscle interface that presents with bruising type of skin discoloration or as a blood-filled blister. ${ }^{2,12}$ The most common PI areas in order of highest to lowest prevalence in older adults are the sacrum, heels, trochanter, and ischium. ${ }^{11}$ These skin sites are important to examine among older patients and PI staging documentation should be completed as soon as possible to guide interventions.

\section{Friction and Shear}

Various PI stages are influenced by the different forces exerted such as friction and shear. Friction is defined as the, "mechanical force exerted when skin is dragged across a coarse surface," and skin damage due to friction alone typically leads to superficial abrasions with vulnerability to PIs. ${ }^{13}$ Shear is a mechanical force that acts internally on the skin tissue in a direction parallel to the body's surface. ${ }^{13-16}$ Shearing forces occur between the internal body structures and skin tissues typically moving in opposite directions and may lead to deep tissue injury. ${ }^{14,16}$ Friction and shear have a direct relationship so if friction increases, then shear likely increases. ${ }^{14}$ Friction forces have the greatest impact on superficial layers of skin via a "top-down" mechanism of tissue injury while shearing forces influence deeper tissue structures via a "bottomup" mechanism of tissue injury. ${ }^{16,17}$ Dynamic conditions like repositioning and patient transfers may drag the skin over bony prominences, increasing both the friction and shearing forces. ${ }^{18,19}$ This increases the risk of superficial tissue injury including skin tears and blister formation particularly in older adults given their skin atrophy, reduced collagen, and loss of elastin. ${ }^{19}$ These movements may also make the skin tissue more vulnerable to deeper PIs.

During friction and shearing forces, tissues are stretched with possible separation of skin layers and blood vessel tearing or twisting leading to impaired blood flow. ${ }^{16,20,21}$ Lack of blood flow that carries oxygen and nutrients to cells causes cell death and results in tissue damage and PI formation. ${ }^{14,20-22}$ Shear stress from 9 to 14.9 kilopascals of force is the threshold range for potential PI formation. ${ }^{23}$ Shearing forces may often exceed this threshold during repositioning tasks such as sliding a patient parallel in bed. ${ }^{3,11}$ Particularly in this scenario, the coccyx bone moves up while the skin is moving down, leading to forces in the opposite direction that increases the likelihood of skin tear and decreased perfusion with PI risk in the deep tissues over the coccyx bone. ${ }^{11}$ Frequent movement leads to differences in temperature and moisture conditions that place the skin at increased vulnerability to injury. The friction coefficient of human skin increases when it is moist and contributes to PI formation. ${ }^{24}$ Therefore, it is important to promote an optimal skin microclimate utilizing appropriate PI risk reduction linens and support surfaces. ${ }^{11,14}$ Friction increases between skin and textiles under wet conditions, so it is important to keep both surfaces clean and dry. ${ }^{25}$

\section{Friction and Shear Risk Reduction}

There are many strategies to help reduce friction and shear. Specific attention to skin care, selection of support surfaces and fabrics, positioning techniques, and use of Safe Patient Handling and Mobility (SPHM) technology among older adults are highlighted here. Approaches to reduce friction and shear include using skin barrier products and avoiding massage during skin care. ${ }^{26}$ Protective products such as cyanoacrylate topical skin protectants increase skin surface stiffness and can reduce the effects of friction forces on thin elderly skin. ${ }^{15}$ Protective dressings may also be used over bony prominences during movement to help prevent skin damage during patient transfers. ${ }^{11}$ The roughness of surfaces underneath patients is also a main contributor to friction and shear especially among older adults with decreased skin elasticity..$^{10}$ Seat cushions comprised of viscous fluid followed by air cell, elastic or viscoelastic foam, and honeycomb were found to have the least interface shear stress. ${ }^{27}$ Synthetic fibers found in low friction textiles were found to have less friction and more water transport abilities than standard hospital sheets. ${ }^{24}$ Thus, selecting proper skin care products and surface textures may help decrease PI injury formation in older adults.

Proper positioning and SPHM technology during patient transfers may also reduce friction and shearing forces that may lead to PI injury in the older population. Friction and shear increase during transfers especially if the patient is lying on an incline or uneven surface. ${ }^{24}$ A supine position with the head of bed elevated over 30 degrees is also known to increase shear given the patient may start to slide downward. ${ }^{4}$ Researchers report backward direction shearing forces occur below the ischial tuberosity even while in a seated position like in a wheelchair. ${ }^{28}$ Furthermore, shear increases with leaning forward and whenever the torso is tilted to one side, which causes the 
shear to increase in the opposite direction. ${ }^{28}$ The use of SPHM technology during patient transfers also warrant considerations to avoid PI risk, given some equipment or related textiles may decrease friction, shear or pressure while others may increase these forces. ${ }^{29} \mathrm{Few}$ examples of SPHM devices include friction-reducing transfer sheets, air transfer devices, transfer boards, and overhead lifting devices. ${ }^{15,26,30}$ Friction-reducing sheets as opposed to standard cotton sheets when used during lateral patient transfers may help reduce skin drag. ${ }^{30}$ Other devices like transfer boards and slings may increase friction along the skin so proper selection of equipment based on patient size, needs, and skin features are imperative. ${ }^{29}$ These techniques may optimize the skin integrity during transfers especially among older adults with vulnerable skin to PI.

Many clinical PI risk assessment tools over the years have acknowledged the potential impact of friction and shearing forces. ${ }^{11}$ For example, the friction and shear component of the Braden Scale for Predicting Pressure Sore Risk asks how much assistance is required to move a patient versus the patient being able to completely lift from a bed or chair independently without sliding. ${ }^{31}$ New innovations have been developed to assist healthcare providers to monitor friction, shearing, and pressure forces. A novel compliant shear force sensor was designed to be placed on the skin and bed or seat surface interface to help early detection of shearing forces to permit intervention. ${ }^{23}$ Real-time pressure-mapping sensors that can be incorporated into bed or seating cushions have also been developed. Some pressure-mapping sensors demonstrate higher areas of pressure in different colors so that repositioning may be optimized..$^{32}$ Thermographic imaging has been used to detect temperature changes in the skin. Areas like the sacrum have been found to have the highest mean temperature which aids thermal detection of PI prone skin, but people 60 years and older may not have as high of a thermal response, making injury detection more challenging by this clinical parameter in the older population. $^{33}$

\section{Conclusion}

Overall, aging skin is particularly susceptible to PI injury due to friction and shearing forces and PI prevention strategies during patient transfers are warranted especially for older adults. Friction mainly contributing to superficial and shear to deeper skin tissue damage typically coincide in most scenarios. Barriers to friction and shear reduction include lack of knowledge of these forces, ability to accurately measure these forces, and appropriate product selection to reduce these forces. ${ }^{4}$ The majority of PI prevention research has targeted pressure force reduction through offloading, but there is growing awareness of friction and shear importance to PI development leading to more studies on how to address these barriers. ${ }^{3,15,16,18}$ The complexities of skin aging with the interactions of these forces make the older population at more risk for PIs. This highlights the need for skin protection during patient care and repositioning, which warrants the inclusion of older adults in future studies on this topic.

\section{Acknowledgement}

The authors wish to acknowledge the Veterans Health Administration VISN 8 Patient Safety Center of Inquiry and the James A. Haley Veterans' Hospital and Clinics for their long history of work in patient safety and safe patient handling. There was no financial funding for this manuscript development. The contents of this manuscript do not represent the views of the Department of Veterans Affairs or the United States Government.

\section{Conflict of Interest}

Both authors report they have no Conflict of Interest related to this work.

\section{References}

1. Russell-Goldman E, Murphy GF. The Pathobiology of Skin Aging: New Insights into an Old Dilemma. American Journal of Pathology. 2020. Volume 190, Issue 7: 1356-1369. ISSN 0002-9440. https://doi. org/10.1016/j.ajpath.2020.03.007

2. Cowan L, Broderick V, Alderden JG. Pressure Injury Prevention Considerations for Older Adults. Crit Care Nurs Clin North Am. 2020 Dec; 32(4): 601-609. doi: 10.1016/j.cnc.2020.08.009. PMID: 33129417.

3. Gillespie BM, Walker RM, Latimer SL, et al., Repositioning for pressure injury prevention in adults. Cochrane Database Syst Rev. 2020 Jun 2; 6(6): CD009958. doi: 10.1002/14651858.CD009958.pub3. PMID: $32484259 ;$ PMCID: PMC7265629.

4. De Wert LA, Bader DL, Oomens CW, et al., A new method to evaluate the effects of shear on the skin. Wound Repair Regen. 2015 Nov-Dec; 23(6): 885-90. doi: 10.1111/wrr.12368. Epub 2015 Nov 4. PMID: 26426393.

5. Dobrev H. Organization of the dermal matrix impacts the biomechanical properties of skin. Br J Dermatol. 2017 Sep; 177(3): 622-623. doi: 10.1111/bjd.15762. PMID: 28940269.

6. Langton AK, Graham HK, McConnell JC, et al., Organization of the dermal matrix impacts the biomechanical properties of skin. Br J Dermatol. 2017 Sep; 177(3): 818-827. doi: 10.1111/bjd.15353. Epub 2017 Jun 12. PMID: 28132410.

7. Langton AK, Graham HK, Griffiths CEM, et al., Ageing significantly impacts the biomechanical function and structural composition of skin. Exp Dermatol. 2019 Aug; 28(8): 981-984. doi: 10.1111/ exd.13980. PMID: 31152614; PMCID: PMC6851988.

8. Payne D. Skin integrity in older adults: pressure-prone, inaccessible areas of the body. Br J Community Nurs. 2020 Jan 2; 25(1): 22-26. doi: 10.12968/bjcn.2020.25.1.22. PMID: 31874079.

9. Blair MJ, Jones JD, Woessner AE, et al., Skin Structure-Function Relationships and the Wound Healing Response to Intrinsic Aging. Adv Wound Care (New Rochelle). 2020 Mar 1; 9(3): 127-143. doi: 10.1089/wound.2019.1021. Epub 2020 Jan 24. PMID: 31993254; PMCID: PMC6985772.

10. Leyva-Mendivil MF, Lengiewicz J, Page A, et al., Skin Microstructure is a Key Contributor to Its Friction Behaviour. Tribol Lett. 2017; 65(1): 12. doi: $10.1007 / \mathrm{s} 11249-016-0794-4$. 
11. Al Aboud AM, Manna B. Wound Pressure Injury Management. 2020 Jun 28. In: StatPearls [Internet]. Treasure Island (FL): StatPearls Publishing; 2020 Jan-. PMID: 30422492.

12. National Pressure Injury Advisory Panel (NPIAP). NPIAP Pressure Injury Stages revised 2016. Available at www.npiap.com and at: https://cdn.ymaws.com/npiap.com/resource/resmgr/online_store/ npiap_pressure_injury_stages.pdf. Accessed May 18, 2021.

13. Hess CT. Did You Know? The Difference between Friction and Shear Advances in Skin \& Wound Care. June 2004; 17(5): 222-224.

14. Gefen $\mathrm{A}$. Why is the heel particularly vulnerable to pressure ulcers? Br J Nurs. 2017 Nov 8; 26(Sup20): S62-S74. doi: 10.12968/bjon.2017.26. Sup20. S62. PMID: 29120686.

15. Gefen A. The bioengineering theory of the key modes of action of a cyanoacrylate liquid skin protectant. IntWound J. 2020; 17: 13961404. https://doi.org/10.1111/iwj.13401.

16. Hoogendoorn I, Reenalda J, Koopman BFJM, et al., The effect of pressure and shear on tissue viability of human skin in relation to the development of pressure ulcers: a systematic review. J Tissue Viability. 2017 Aug; 26(3): 157-171. doi: 10.1016/j.jtv.2017.04.003. Epub 2017 Apr 14. PMID: 28457615.

17. Stechmiller JK, Cowan LJ, Oomens CWJ. Bottom Up (Pressure Shear) Injuries (Chapter 18), in Wound, Ostomy and Continence Nurses Society Core Curriculum: Wound Management, 1st edition, edited by Dorothy B. Doughty and Laurie McNichol. May 2015. Wolters Kluwer Health and Lippincott Williams \& Wilkins.

18. Call E, Tanner L, Cheney A, et al., Results of Laboratory Testing for Immersion, Envelopment, and Horizontal Stiffness on Turn and Position Devices to Manage Pressure Injury, Advances in Skin \& Wound Care. October 2020. Volume 33, Issue 10S: S11-S22. doi: 10.1097/01.ASW.0000696412.04000.98.

19. Moda Vitoriano Budri A, Moore Z, Patton D, et al., Impaired mobility and pressure ulcer development in older adults: Excess movement and too little movement-Two sides of the one coin? J Clin Nurs. 2020 Aug; 29(15-16): 2927-2944. doi: 10.1111/jocn.15316. Epub 2020 May 25. PMID: 32380572.

20. Manorama A, Baek S, Vorro J, et al., Blood perfusion and transcutaneous oxygen level characterizations in human skin with changes in normal and shear loads--implications for pressure ulcer formation. Clin Biomech (Bristol, Avon). 2010 Oct; 25(8): 823-8. doi: 10.1016/j. clinbiomech.2010.06.003. PMID: 20663594

21. Manorama A, Meyer R, Wiseman R, et al., Quantifying the effects of external shear loads on arterial and venous blood flow: implications for pressure ulcer development. Clin Biomech (Bristol, Avon). 2013 Jun; 28(5): 574-8. doi: 10.1016/j.clinbiomech.2013.04.001. Epub 2013 Apr 20. PMID: 23611582.
22. Leung IPH, Fleming L, Walton L, et al., Development of a model to demonstrate the effects of friction and pressure on skin in relation to pressure ulcer formation. Wear. 2017. Vol. 376-377: 266-271. ISSN 0043-1648. https://doi.org/10.1016/j.wear.2016.11.026.

23. Chaykina A, Griebel S, Zentner L. Design, fabrication, and characterization of a compliant shear force sensor for a humanmachine interface. Sensors and Actuators A: Physical. 2016. Vol 246: 91-101. ISSN 0924-4247.https://doi.org/10.1016/j.sna.2016.04.034.

24. Derler S, Rao A, Ballistreri P, et al., Medical textiles with low friction for decubitus prevention. Tribology International. 2012. Volume 46, Issue 1:208-214. ISSN 0301-679X. https://doi.org/10.1016/j. triboint.2011.03.011

25. Rotaru GM, Pille D, Lehmeier FK, et al., Friction between human skin and medical textiles for decubitus prevention. Tribology International. 2013. Vol 65: 91-96. ISSN 0301-679X. https://doi.org/10.1016/j. triboint.2013.02.005

26. Yilmazer T, Inkaya B, Tuzer H. Care under the guidance of pressure injury prevention protocol: a nursing home sample. Br J Community Nurs. 2019 Dec 1; 24(Sup12): S26-S33. doi: 10.12968/bjcn.2019.24. Sup12. S26. PMID: 31804884.

27. Akins IS, Karg PE, Brienza DM. Interface shear and pressure characteristics of wheelchair seat cushions. J Rehabil Res Dev. 2011; 48(3): 225-34. doi: 10.1682/jrrd.2009.09.0145. PMID: 21480097.

28. Shirogane S, Toyama S, Takashima A, et al., The relationship between torso inclination and the shearing force of the buttocks while seated in a wheelchair: Preliminary research in non-disabled individuals. Assist Technol. 2020 Nov 1; 32(6): 287-293. doi:10.1080/10400435. 2018.1547333. Epub 2018 Nov 30. PMID: 30500299.

29. Peterson MJ, Kahn JA, Kerrigan MV, et al., Pressure ulcer risk of patient handling sling use. J Rehabil Res Dev. 2015; 52(3): 291-300. doi: 10.1682/JRRD.2014.06.0140. PMID: 26237005

30. Hwang J, Kuppam VA, Chodraju SSR, et al., Commercially Available Friction-Reducing Patient-Transfer Devices Reduce Biomechanical Stresses on Caregivers' Upper Extremities and Low Back. Hum Factors. 2019 Nov; 61(7): 1125-1140. doi: 10.1177/0018720819827208. Epub 2019 Feb 22. PMID: 30794442.

31. Bergstrom N, Braden BJ, Laguzza A, Holman V. The Braden Scale for Predicting Pressure Sore Risk. Nurs Res. 1987 Jul-Aug;36(4):205-10. PMID: 3299278.

32. Behrendt R, Ghaznavi AM, Mahan M, et al., Continuous bedside pressure mapping and rates of hospital-associated pressure ulcers in a medical intensive care unit. Am J Crit Care. 2014 Mar; 23(2): 127-33. doi: 10.4037/ajcc2014192. PMID: 24585161.

33. Soares RS, Lima SB, Eberhardt TD, et al., Skin temperature as a clinical parameter for nursing care: a descriptive correlational study. J Wound Care. 2019 Dec 2; 28(12): 835-841. doi: 10.12968/ jowc.2019.28.12.835. PMID: 31825777. 